\title{
Resource Competition Determines Selection of B Cell Repertoires
}

\author{
Rob J. De Boer*†, António A. Freitasł and Alan S. Perelson§ \\ *Theoretical Biology, Utrecht Univerity, Padualaan 8, 3584 CH Utrecht, The Netherlands, \$Unité \\ Biologie des Populations Lymphocytaires, CNRS URA 1961, Institut Pasteur, Paris, France and \\ §Theoretical Division, MS-K710, Los Alamos National Laboratory, Los Alamos, NM 87545, U.S.A.
}

(Received on 20 December 2000, Accepted in revised form on 20 June 2001)

\begin{abstract}
Previous experiments with mouse chimeras demonstrated that cellular competition for antigen-specific survival signals plays a crucial role in the maintenance of the naive B cell repertoire. Transgenic (Tg) B cell populations in these chimeras have a shortened lifespan and poor competitive abilities as compared to more diverse non-Tg populations in the same mice. We develop a mathematical model to investigate the mechanism of B cell competition. The model allows for various B cell clones, generated in the bone marrow, to go into the peripheral circulation, where they compete specifically for various ligands providing survival signals. In the model we also find the observed poor competitive abilities of the Tg repertoire. Investigating the nature of the competition in the model, we find that most of the competition is "intraspecific" occurring largely within the clone of truly Tg B cells, and within the repertoire of leaky Tg and non-Tg B cells. This is confirmed by analysing a simplified version of the model, which only allows for intraspecific competition, and resembles a simple ecological model with density-dependent death. The fact that our model accounts for the data, casts doubt on a previous interpretation of the same data arguing that more diverse repertoires outcompete repertoires of lower diversity. Here, we conclude that most of the data can be explained with intraspecific competition, and formulate an experimental prediction that allows one to distinguish between the previous interpretation of inter-specific competition between repertoires, and the current interpretation of intraspecific competition.
\end{abstract}

(C) 2001 Academic Press

\section{Introduction}

The maintenance of the peripheral pools of $\mathrm{T}$ and $\mathrm{B}$ lymphocytes is an active homeostatic process of lymphocytes competing for survival and renewal signals (Rocha et al., 1989; Freitas \& Rocha 2000). $\mathrm{T}$ and $\mathrm{B}$ cell repertoires are partly maintained by de novo production of mature cells in the thymus and bone marrow (Freitas \& Rocha 1993; Tanchot \& Rocha, 1995,

\footnotetext{
* Author to whom correspondence should be addressed. E-mail:r.j.deboer@bio.uu.nl.
}

1997; Mackall \& Gress, 1997), and partly by competitive renewal in the periphery (Rocha et al., 1989; Freitas \& Rocha, 1993; Mackall et al., 1996, 1997). For T cells this has recently been called "homeostatic proliferation" (Viret et al., 1999; Kieper \& Jameson, 1999; Ernst et al., 1999; Surh \& Sprent, 2000).

The naive $\mathrm{B}$ cell repertoire is largely maintained by de novo production of mature cells in the bone marrow (Freitas \& Rocha, 1993; Freitas et al., 1995; Agenes et al., 1997; Freitas \& Rocha, 2000). The bone marrow has an overproduction because about one-third of the normal number of 
pre-B cells is sufficient for maintaining a normal peripheral B cell pool size (Agenes et al., 1997). Similarly, parabiosis experiments show that the $B$ cell production of one mouse suffices for maintaining normal peripheral B cell numbers in at least three mice (Agenes et al., 1997) The de novo production of naive $B$ cells in the bone marrow (Agenes et al., 1997) seems independent of the peripheral B cell population size. Thus, the naive $\mathrm{B}$ cell repertoire appears to be under homeostatic control where the peripheral survival rate depends on the population density (Freitas et al., 1995; Agenes et al., 1997; Freitas \& Rocha, 2000). In this manner, density-dependent peripheral survival rates can compensate for differences in bone marrow production, which accounts for the phenomenon of homeostasis. Thus, there is no typical "pre-programmed" lymphocyte lifespan (Freitas \& Rocha, 1993; Tanchot \& Rocha, 1997, 1998), because the death rate is at least partly determined by the total lymphocyte density.

A major question in lymphocyte homeostasis is whether competition between lymphocytes involves ligands (resources) specifically stimulating the antigen receptor. We have previously developed models for competition in $\mathrm{T}$ cell repertoires, and have shown how the diversity of the repertoire depends on the diversity of resources the clones in the repertoire are competing for (De Boer \& Perelson, 1997). For naive T cells it is relatively well established that TCR/MHC interactions are essential for survival (Witherden et al., 2000). Naive B cell survival also requires the $\mathrm{BCR}$ because $\mathrm{B}$ cells lacking the V-region of the IgM receptor have a very short lifespan (Rosado \& Freitas, 1998). The fact that the BCR is required for B cell survival does not necessarily imply that $\mathrm{B}$ cells are competing for antigen-specific signals, however. It could be that the lifespan of the $\mathrm{B}$ cells that are rescued by tickling the BCR remains determined by competition for non-specific resources like lymphokines, adhesion molecules, and/or "space" in the lymphoid tissue. Previous in vivo experiments with transgenic mice, however, demonstrated that competition for antigen-specific resources does play a crucial role in the survival of naive B cells (Freitas et al., 1995) [and also of CD8 ${ }^{+}$ T cells (Freitas et al., 1996)]. We will here develop a mathematical model to improve the interpretation of these fairly complicated experimental results.

Irradiated recipient mice reconstituted with mixtures of bone marrow cells from different donor mice showed that the survival time of the B lymphocytes from one donor depends on the presence, and the repertoire diversity, of the other donor (Freitas et al., 1995). In these experiments lethally irradiated mice were reconstituted with normal and/or transgenic bone marrow cells. Even allowing for the usual "leakiness" of the transgenic $(\mathrm{Tg}) \mathrm{B}$ cells, the immunoglobulin diversity of the $\mathrm{Tg}$ repertoire should be considerably smaller than that of a non-Tg repertoire. Nevertheless, mice reconstituted with $100 \% \mathrm{Tg}$ bone marrow cells obtain approximately normal steady-state total B cell numbers. Mice reconstituted with mixtures of non- $\mathrm{Tg}$ and $\mathrm{Tg}$ bone marrow cells in different ratios, always had more than a proportional number of non-Tg B cells in their peripheral repertoire (Freitas et al., 1995). Very similar results were obtained with parabiosis experiments with normal and transgenic mice (Agenes et al., 1997). Thus, increasing B cell repertoire diversity seems to increase the competitive ability of the repertoire as a whole. This strongly suggests that antigen-specific competition is involved in the homeostasis of naive B cell repertoires.

The data in Freitas et al. (1995) have previously been analysed with a mathematical model describing the non-Tg and the Tg repertoire as two competing (meta) populations (McLean et al., 1997). Varying the parameter describing the competition between the Tg repertoire and the non-Tg repertoire, this model was able to fit the data. This result was therefore interpreted to mean that a more diverse repertoire could outcompete a less diverse repertoire (McLean et al., 1997).

Antigen-specific competition functions at the level of the individual clones, however, and not at the level of whole repertoires. Thus, we here develop a new model that explicitly considers the individual B cell clones in the non-Tg and the Tg repertoire, and a variety of ligands these clones are competing for. Summing over all the individual clones within the non- $\mathrm{Tg}$ and in the $\mathrm{Tg}$ repertoire, we obtain data from our model that 
also fit the experimental data of Freitas et al. (1995), and provide an alternative explanation for the preferential survival of the non-Tg B cells when placed in competition with $\mathrm{Tg} \mathrm{B}$ cells. We find that "intraspecific" competition within the Tg-repertoire is sufficient to explain the data.

\section{Methods}

We mathematically model the data presented by Freitas et al. (1995). Lethally irradiated (850-900 rads) host mice (8 week, female, C57BL/6, Iffa Credo, France) received i.v. a total of $4 \times 10^{6}$ bone marrow cells from donor mice mixed at different ratios. The mice were kept in specific pathogen-free conditions; most B cells in the repertoire should therefore be naive. The donor mice were "non-Tg" C57BL/6 IgH"; "MD4", a C57BL/6 line expressing a transgene coding for the $\mu^{a}+\delta^{a}$ heavy and light chains of an antibody to hen egg-white lysozyme (HEL) (Goodnow et al., 1988); and "SP6", a C57BL/6 line expressing a transgene coding both $\mu^{a}$ heavy and light chains of a complete BALB/c antibody to 2,4,6trinitrophenol (TNP) (Rusconi \& Kohler, 1985). According to previous experiments, these populations could be ranked linearly by competitive ability as non-Tg > MD4 > SP6 (Freitas et al., 1995). For the BrdU labeling, mouse chimeras were injected i.p. with $1 \mathrm{mg}$ of BrdU every $12 \mathrm{~h}$ for 1,3 or 6 consecutive days. Purified bone marrow and spleen cells from BrdU-injected chimeras were detected according to published protocols (Freitas et al., 1995).

\section{Model}

Consider an immune repertoire composed of many naive $\mathrm{B}$ cell clones $B_{i}$. Each clone is maintained by a source $m_{i}$ of specific cells from the bone marrow. For simplicity, we ignore the relatively small contribution of naive B cell renewal, i.e. the naive $B$ cells in our model do not divide. The average lifespan of any $\mathrm{B}$ cell in a repertoire is determined by interactions with "antigenspecific survival signals", here called "epitopes". Competition for survival signals comes about when specific $B$ cells decrease the epitope availability by temporarily binding with the epitopes.
B cells receiving sufficient survival signals have a slow death rate $d$; B cells receiving fewer survival signals are assumed to have higher death rates.

Since all the B cells of one particular clone $B_{i}$ have the same specificity, they have to interact with the same set of epitopes in order to obtain survival signals. The niche overlap between the cells of a clone is therefore always larger than that with cells of other clones. This is typical for resource competition: the within-clone, i.e. "intraspecific", competition is always stronger than the "inter-specific" competition between clones of different specificities.

B cell clones can be specific for a number, say $c$ (for "connectivity"), of different epitopes, and hence compete with all other clones having a specificity for any of these epitopes. Let there be $e$ epitopes and $n \mathrm{~B}$ cell clones in the model. Since the concentrations and the affinities of epitopes providing survival signals are not known, we scale their concentrations to one and treat the affinities as fixed random parameters between zero and one. The information on who interacts with whom can therefore be gathered in an $n \times e$ interaction matrix $K$, with elements $0 \leqslant K_{i j}<1$, in which each row lists the interactions of one particular clone. Since there is no information on the topology of the interaction of the B cell repertoire with the epitopes maintaining it, we let this matrix be random. Other topologies are not expected to affect the results. On average each row in the matrix has $c$ non-zero elements for the affinities of the (randomly chosen) epitopes the clone interacts with.

When cells compete for epitopes, the net availability of each epitope $j$ at equilibrium is

$$
F_{j}=\frac{1}{1+\sum_{i}^{n} K_{i j} B_{i}},
$$

where $B_{i}$ is the density of B cell population $i$. In the absence of $\mathrm{B}$ cells each epitope has a scaled availability of $F_{j}=1$. Increasing B cell numbers in the denominator decreases the epitope availability. Equation (1) has been derived previously in models describing the binding of lymphocytes to ligands (De Boer \& Perelson, 1994, 1995, 1997).

The total survival signal to a clone $i$ is the sum over all affinity and availability weighted 
epitopes, $F_{j}$, i.e.

$$
S_{i}=\sum_{j=1}^{e} K_{i j} F_{j} .
$$

The B cell clones, $B_{i}$, are maintained by generation from the bone marrow at rate $m_{i}$, and have a density-dependent survival rate that is determined by the clonal survival signal $S_{i}$. Assuming that the rate of cell loss is inversely related to the survival signal we write that

$$
\frac{\mathrm{d} B_{i}}{\mathrm{~d} t}=m_{i}-\frac{d c}{2 S_{i}} B_{i}
$$

where $m_{i}$ defines the clonal source from the bone marrow, and $d$ is the death rate constant in the absence of competition. The death rate constant is multiplied by a factor $c / 2$ to make the average loss rate of a clone in the absence of competition independent of the connectivity parameter $c$. In the absence of competition $F_{j}=1$, and each clone is expected to receive a total stimulus of $S_{j}=c \hat{K}$, where $\hat{K}=0.5$ is the average interaction strength. The precise form of the competition function in eqn (3) is not expected to affect the results since we have obtained very similar behavior with models where the antigen-specific competition term was regulating clonal renewal rates (De Boer \& Perelson, 1997).

In the model we consider three categories of $\mathrm{B}$ cells differentiating from $\mathrm{Tg}$ and from non- $\mathrm{Tg}$ bone marrow progenitors: (i) truly $\mathrm{Tg} \mathrm{B}$ cells, expressing the antigen receptor encoded by the transgene only, (ii) leaky-Tg B cells, expressing the $\mathrm{Tg}$ antigen receptor plus a randomly rearranged new one, and (iii) non- $\mathrm{Tg} \mathrm{B}$ cells, expressing a randomly rearranged antigen receptor. After reconstitution, let $P_{T}$ be the fraction of transgenic $\mathrm{B}$ cell precursors in the bone marrow, and let $P_{L}$ be a "leakiness" parameter denoting the fraction of transgenic bone marrow cells producing B cells expressing both a random receptor and the Tg receptor. Thus, the fraction of leakyTg B cells is $P_{T} P_{L}$, and the fraction of truly Tg precursors in the bone marrow is $P_{T}\left(1-P_{L}\right)$.

The diversity of the non-Tg and the leaky-Tg repertoires should be proportional to the corresponding number of bone marrow precursors. If we consider a bone marrow consisting of $M$ bone marrow cells, then we expect $n_{l}=P_{L} P_{T} M$ leakyTg and $n_{n}=\left(1-P_{T}\right) M$ non-Tg clones, and one truly Tg clone with $\left(1-P_{L}\right) P_{T} M$ bone marrow precursors. Thus, the total diversity of the system, $n=1+n_{l}+n_{n}$, varies between $n=M$ in a completely non-Tg system (where $P_{T}=0$ ) and $n=1+P_{L} M$ in a fully transgenic system (where $\left.P_{T}=1\right)$. The parameters $M, P_{T}$, and $P_{L}$ thus determine the diversity $n$ in our model. The total diversity of the model repertoire should be so large that it does not limit the total number of B cells (see De Boer \& Perelson, 1997). We have tested the robustness of our results by allowing for a higher diversity by increasing $M$ ten-fold. If each bone marrow cell produces one cell per day then the source rate of new cells entering the periphery $m_{i}=\left(1-P_{L}\right) P_{T} M$ cells/day for the truly Tg clone. Further, if we assume that each non-Tg and leaky-Tg bone marrow cell produces B cells with different specificities, then $m_{i}=1$ cell/day for each such specificity.

The model was coded in the $\mathrm{C}$ programming language, and the equations were solved using a Runge-Kutta variable time step integration routine (Press et al., 1988). We only depict steadystate results, i.e. for any parameter setting the system was run until the unique state state was approached (see the appendix).

\section{Results and Discussion}

TOTAL B CELL NUMBERS

The experimental data on the competition between the repertoires of non- $\mathrm{Tg} B$ cells and those derived from the transgenic MD4 or SP6 bone marrow cells are summarized in Fig. 1. In both experiments the total B cell numbers in the spleen (see the circles in Fig. 1) increase with the fraction of non-Tg cells. In the spleen the fraction of $\mathrm{Tg}$ $\mathrm{B}$ cells is always lower than that in the inoculum (or that in the bone marrow) (Freitas et al., 1995). Thus, on average, non-Tg B cells are positively selected, demonstrating the superior resource competition of the non-Tg repertoire, and leading to the counterselection of $\mathrm{Tg} \mathrm{B}$ cells. In the double transgenic chimeras of MD4 with SP6 cells one finds that the MD4 repertoire is preferentially selected (Freitas et al., 1995). This competitive superiority of the MD4 repertoire is reflected in Fig. 1 by the higher fractions of $\mathrm{Tg}$ 

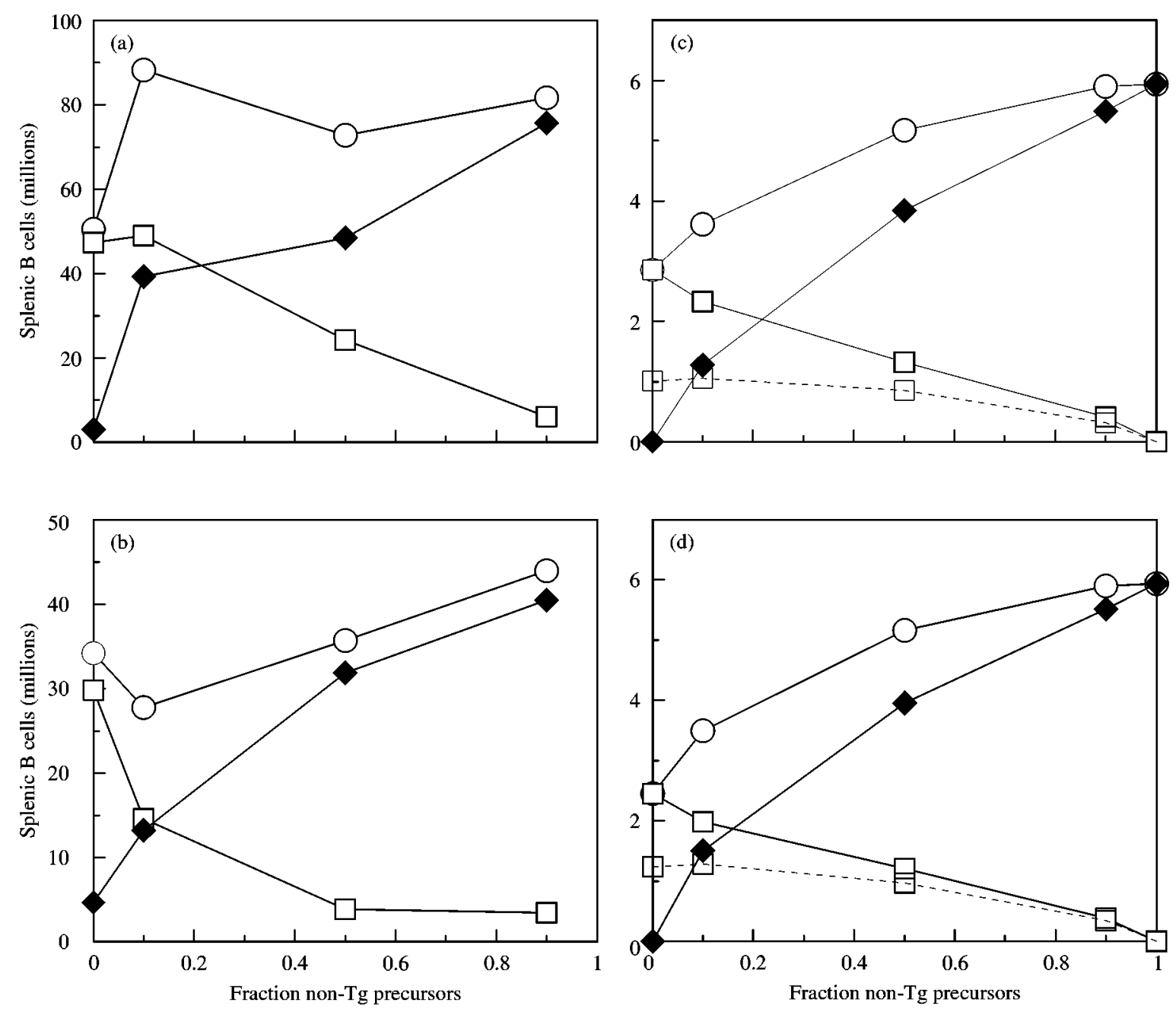

FIG 1. A comparison of the model behavior with the competition data as provided by Table 1 in Freitas et al. (1995). Panels (a) and (b) depict the data in millions of B cells per spleen as a function of the fraction of non-Tg bone marrow cells the mice were reconstituted with. Total B cell numbers are indicated by the circles and the numbers of non-Tg B cells are indicated by black diamonds. The numbers of splenic Tg B cells, i.e. MD4 in panel (a) and SP6 in panel (b), are indicated by the squares. Due to their superior competition the fraction of non- Tg B cells in the spleen is always larger than the fraction of non-Tg cells in the bone marrow inoculum. Total B cells increase somewhat with increasing fractions of non-Tg B cells. Panels (c) and (d) depict similar data from the mathematical model for a leakiness of $10 \%$ (c), and $5 \%$ (d), respectively. The dashed line depicts the total number of truly transgenic B cells. Total B cell numbers in panels (c) and (d) are steady-state numbers (as attained by computer simulation) and were divided by $M$ to normalize to an average population size. Parameters $M=500$ bone marrow cells, $c=10$ connections, $e=100$ epitopes, $d=10^{-3} /$ day, and $P_{L}=0.1$ in (c) and $P_{L}=0.05$ in (d). For leaky-Tg and non-Tg clones $m_{i}=1 \mathrm{~B}$ cell/day, and for the truly Tg clone $m_{i}=\left(1-P_{L}\right) P_{T} M$ cells/day.

B cells in Fig. 1(a), depicting a non-Tg/MD4 chimera, as compared to those in Fig. 1(b), depicting a non- $\mathrm{Tg} / \mathrm{SP} 6$ chimera.

The steady-state behavior of our mathematical model [see Fig. 1(c) and (d)] can be compared with the data [in Fig. 1(a) and (b)], by changing the fraction $P_{T}$ of $\mathrm{Tg}$ cells in the model bone marrow. In our model the size of the Tg population is in arbitrary units, and is the sum of the size of the truly $\mathrm{Tg}$ clone and all leaky-Tg clones. Similarly, the size of the non-Tg repertoire is the sum of the clone sizes of all non-Tg clones. Setting the leakiness parameter, $P_{L}$, to $10 \%$, we obtain ratios of total transgenic and non- $\mathrm{Tg}$ $B$ cells that are qualitatively similar to those of the MD4 data [see Fig. 1(c)]. The main difference between the model and the data is the somewhat larger increase in total $\mathrm{B}$ cell numbers in the 
model when the fraction of non-Tg precursors is increased. In comparative models, the steadystate total number of cells is determined by the total number of resources (i.e. epitopes) being exploited by the repertoire as a whole (De Boer $\&$ Perelson, 1997). Increasing the repertoire diversity by increasing the fraction of non- $\mathrm{Tg}$ cells in the bone marrow, should therefore increase total B cell numbers. This effect should disappear when there exists an additional non-specific regulation of B cell survival rates, and/or when the diversity of the $\mathrm{Tg}$ repertoire is so large that it maximally exploits all epitopes (i.e. when the parameter $M$ is set sufficiently large).

The data obtained in the non- $\mathrm{Tg} / \mathrm{SP} 6$ chimera are qualitatively similar to those of the non$\mathrm{Tg} / \mathrm{MD} 4$ chimera. Both experiments can therefore be accounted for with this model. One way to achieve this is to assume that the diversity of the functional SP6 repertoire is somewhat lower than that of the MD4 repertoire. In our model this can be implemented by reducing the leakiness to $5 \%$ [see Fig. 1(d)]. This allows the Tg MD4 repertoire to recognize a wider diversity of ligands than the Tg SP6 repertoire, i.e. to have a wider variety of niches.

Our model allows us to dissect the Tg repertoire into the truly $\mathrm{Tg}$ clone and the leaky- Tg clones. In Fig. 1(c) and (d) the dashed line depicts the total size of the truly Tg clone. The truly Tg clone attains its maximum size when the fraction of $\mathrm{Tg}$ precursors in the bone marrow is maximal. This was expected because increasing the number of $\mathrm{Tg}$ bone marrow cells increases the source of truly Tg B cells. Since the truly Tg B cells are all competing for the same set of ligands this is, however, also expected to intensify their competition for survival signals, which should be reflected in increased death rates at high $\mathrm{Tg}$ ratios. Indeed similar results were found in recent experiments with $\mathrm{RAG}^{-1-}$ mice in which non-Tg B cells compete with monoclonal MD4 Tg B cells (Freitas, work in progress).

\section{DEATH RATES}

In Freitas et al. (1995) the turnover in the non-Tg and the $\mathrm{Tg}$ repertoires was studied by BrdU labeling. Mice reconstituted with non- $\mathrm{Tg}$ and $\mathrm{Tg}$ cells were given repeated injections of $\mathrm{BrdU}$, which is incorporated in dividing cells in both the bone marrow and the periphery. In mice with mixtures of bone marrow cells containing $10 \%$ non-Tg cells, after 6 days of BrdU administration, $10 \%$ of the non-Tg splenic B cells, $20 \%$ of the SP6-Tg splenic B cells, and $25 \%$ of the MD4-Tg splenic B cells were labeled with BrdU. Thus, in the presence of non- $\mathrm{Tg}$ cells, Tg B cells had an least two-fold higher turnover than the non-Tg B cells. In double $\mathrm{Tg}$ chimeras, reconstituted with 50\% MD4 and 50\% SP6 bone marrow cells, $12 \%$ of both the SP6 and the MD4 spleen cells were labeled with $\mathrm{BrdU}$ at day six (Freitas et al., 1995). Considering that total B cell numbers are approximately at steady state, implying that cell division is approximately balanced by death, these BrdU data argue for higher death rates of Tg B cells in the presence of non- $\mathrm{Tg}$ B cells.

Comparing the average $\mathrm{Tg} \mathrm{B}$ cell death rate (diamonds) with those of the non-Tg B cells (triangles), the results of our model are very similar to the BrdU data (see Fig. 2). At $10 \%$ non-Tg cells in the inoculum, we find a four-fold higher death rate in the Tg compartment. At $90 \%$ non$\mathrm{Tg}$ precursors, the average death rate of $\mathrm{Tg}$ $B$ cells has decreased, and that of non-Tg increased, such that the difference is much smaller. The short average lifespan of Tg B cells is entirely due to the rapid death rates of the truly Tg cells: on average the leaky-Tg B cells live somewhat longer than the non-Tg B cells (see Fig. 2). Additionally, the decrease in the death rate of $\mathrm{Tg}$ $B$ cells when the fraction of non-Tg cells in the inoculum increases is largely due to the truly $\mathrm{Tg}$ B cells (circles in Fig. 2).

These results suggest that the major effect of increasing the fraction of $\mathrm{Tg}$ cells in the bone marrow is increasing the death rate of truly $\mathrm{Tg}$ $\mathrm{B}$ cells. This is confirmed by parabiosis experiments (Agenes et al., 1997). Increasing the fraction of $\mathrm{Tg}$ bone marrow cells increases the source of truly-Tg B cells, which intensifies the withinclone competition between truly $\mathrm{Tg}$ cells for their survival signals. In Fig. 7 of Freitas et al. (1995) we indeed show that in some cases in the lowest fraction of $\mathrm{Tg}$ precursors, there are more than expected $\mathrm{Tg} B$ cells in the periphery. The increase in the average death rate of non-Tg B cells seen in Fig. 2 is due to a similar mechanism. Increasing 


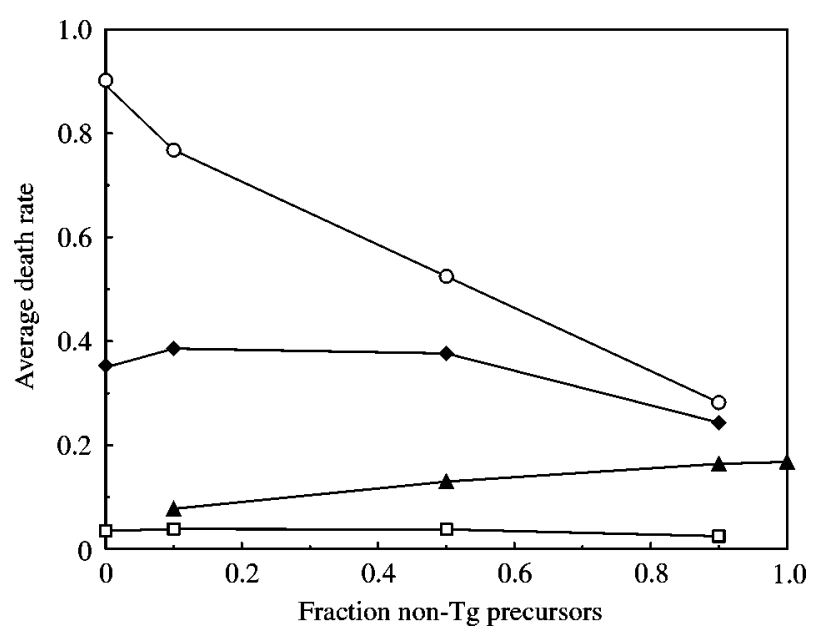

FIG 2. Average cellular death rates in the model. We compute average death rates by calculating at steady state $\left(\sum 0.5 d B_{i} c / S_{i}\right) / \sum B_{i}$ over all classes of Tg and non-Tg B cells; the average death rate of (circles) truly $\mathrm{Tg} B$ cells, (squares) leaky-Tg B cells, (black diamonds) all Tg B cells, and (black triangles) non-Tg B cells. The main effect of increasing the fraction of non- $\mathrm{Tg}$ bone marrow is to decrease the death rate of the truly Tg B cells. Parameters as in Fig. 1 with $P_{L}=0.1$. Note that the result that leaky-Tg B cells have somewhat longer lifespans than non-TG B cells need not be realistic, because one could argue that leaky-Tg B cells, expressing the same total number of BcRs as non-Tg B cells, should have a somewhat lower avidity per ligand. $(\bigcirc-\bigcirc)$ truly- $\mathrm{Tg}$, $(\square-\square)$ leaky-Tg; $\gg$ total-Tg; $\boldsymbol{\Delta}-\boldsymbol{\Delta}$ non-Tg.

the total bone marrow source of non-Tg B cells, also intensifies the (more diffuse) competition amongst the non-Tg cells, leading to somewhat higher death rates.

Thus, the experimental fact that $\mathrm{Tg}$ cells at a $50 / 50 \mathrm{Tg} / \mathrm{Tg}$ ratio had a lower death rate than $\mathrm{Tg}$ cells at a 90/10 Tg/non- $\mathrm{Tg}$ ratio (Freitas et al., 1995), need not imply that Tg B cells live shorter due to the presence of non-Tg B cells. In the 50/50 $\mathrm{Tg} / \mathrm{Tg}$ experiment each $\mathrm{Tg}$ population has a smaller total bone marrow source than the $\mathrm{Tg}$ population in the $90 / 10 \mathrm{Tg} /$ non- $\mathrm{Tg}$ experiments. Hence, slower death rates of Tg B cells were to be expected in these $\mathrm{Tg} /$ non- $\mathrm{Tg}$ chimeras. Below this interpretation is reformulated into a testable experimental prediction.

Summarizing, the mathematical model suggests a novel mechanism for poor competitive ability of $\mathrm{Tg} \mathrm{B}$ cells: we no longer need to invoke much competition between the non- $\mathrm{Tg}$ and the $\mathrm{Tg}$ repertoire to explain the data. The major effect of increasing the fraction of $\mathrm{Tg}$ bone marrow cells is increased in the Tg B cell numbers. By intraspecific competition this increases the death ates of truly Tg B cells. This is investigated further below.

\section{MEAN FIELD MODEL}

Since B cells are competing for resources (Freitas \& Rocha, 2000), it seems only natural that most of the competition in the truly Tg B cell clone is with the other truly $\mathrm{Tg}$ cells of this clone. To obtain survival signals, all B cells from the truly Tg clone have to interact with the same set of epitopes. Moreover, if the truly $\mathrm{Tg}$ clone is very large, the majority of the B cells that are competing for this particular set of epitopes are expected to be truly Tg B cells. This would imply that most of the competition amongst the truly $\mathrm{Tg}$ cells occurs largely within the clone of truly Tg B cells, i.e., is intraspecific. In a model developed below we implement intraspecific competition only, and show that the model can account for the experimental data.

Since truly Tg B cells interact with a subset of the epitopes only, we expect little influence of truly Tg B cells on the survival rates of leaky-Tg and non-Tg B cells. Most of the epitopes maintaining the survival of non-Tg and leaky-Tg $\mathrm{B}$ cells are not recognized by the truly Tg B cells. Hence most of the competition amongst non- $\mathrm{Tg}$ and leaky-Tg B cells is with other non-Tg and leaky-Tg B cells. In the model developed below we therefore combine the leaky-Tg with the nonTg B cell clones into one "diverse" repertoire in which most of the competition is again intraspecific.

This verbal argument is investigated by simplifying our model. Considering the diverse repertoire of leaky-Tg and non-Tg B cells as a single population, and considering one clone of truly $\mathrm{Tg}$ cells, we obtain a model with two differential equations. First, consider the total number of B cells in the diverse repertoire of $n_{n}+n_{l}$ non- Tg and leaky-Tg B cell clones. Clones in this diverse repertoire have an identical source from the bone marrow, an average affinity $K=0.5$, and an average of $c$ interactions in a set of $e$ epitopes. Since each of the $n_{n}+n_{l}$ clones has probability 
$c / e$ to interact with any particular epitope, the average epitope availability is

$$
\hat{F}_{j}=\frac{1}{1+0.5 B c / e}
$$

and hence

$$
\widehat{S}_{i}=0.5 c \widehat{F}_{j}=\frac{c}{2+B c / e}
$$

where $B$ is the total number of $B$ cells in the diverse repertoire of non- $\mathrm{Tg}$ and leaky- $\mathrm{Tg} \mathrm{B}$ cells. Comparing with Eq. (1), one sees that the competition with truly Tg cells has been ignored. The average survival signal $\hat{S}_{i}$ decreases as a function of the total number of $\mathrm{B}$ cells in the diverse repertoire. Equation (3) still represents the dynamics of a single B cell clone in this repertoire, but is now identical for each clone, and can hence be multiplied with $n_{n}+n_{l}$ to obtain an equation for the total number of non-Tg and leaky-Tg $\mathrm{B}$ cells in the repertoire. This gives

$$
\frac{\mathrm{d} B}{\mathrm{~d} t}=n_{n}+n_{l}-\frac{d c}{2 \widehat{S}_{i}} B=n_{n}+n_{l}-\mathrm{d} B(1+0.5 B c / e)
$$

where $n_{n}+n_{l}$ is the total source from the bone marrow. The last term reflects a conventional combination of normal death with densitydependent death. Note that whenever $B \gg e / c$ the density-dependent death dominates over the normal death. For our parameter values this is typically the case (see the appendix).

One can make a similar approximation for the truly $\mathrm{Tg}$ clone by assuming that most of the competition is within the truly Tg population. Each ligand $F_{j}$ that is recognized by the truly $\mathrm{Tg} \mathrm{B}$ cells, $B_{0}$, is interacting with all of them, i.e.

$$
\widehat{F}_{j}=\frac{1}{1+0.5 B_{0}}
$$

and hence

$$
\widehat{S}_{0}=0.5 c \hat{F}_{j}=\frac{c}{2+B_{0}}
$$

where $B_{0}$ is the population size of the truly $\mathrm{Tg}$ clone. This gives

$$
\begin{aligned}
\frac{\mathrm{d} B_{0}}{\mathrm{~d} t} & =M-n_{n}-n_{l}-\frac{d c}{2 \hat{S}_{0}} B_{0} \\
& =M-n_{n}-n_{l}-\mathrm{d} B_{0}\left(1+0.5 B_{0}\right) .
\end{aligned}
$$

Note that the truly $\mathrm{Tg}$ source of $M-n_{n}-n_{l}$ $\mathrm{B}$ cells/day is identical to the source used above of $P_{T}\left(1-P_{L}\right) M$ B cells/day.

Equations (5) and (7) form a two-dimensional caricature model of the combined total non- $\mathrm{Tg}$ and leaky-Tg population $B$, and the truly $\mathrm{Tg}$ clone $B_{0}$. The form of the two equations is a source term, a linear death rate, and a nonlinear density-dependent death rate, which is entirely due to intraspecific competition. By assumption, this model has no inter-specific competition between the truly $\mathrm{Tg}$ clone $B_{0}$ and the population $B$ of non-Tg and leaky-Tg clones, i.e. there is no interaction between the two populations. Since leaky-Tg and non-Tg B cells only interact with a subset of all epitopes, i.e. because $c / e<1$ in eqn (5), such B cells have less intraspecific competition than the truly $\mathrm{Tg} \mathrm{B}$ cells in eqn (7). The truly Tg clone may nevertheless, be maintained at high population numbers when its source $M-n_{n}-n_{l}$ is larger than the source $n_{n}+n_{l}$ of the non-Tg/leaky-Tg cells. Due to the bone marrow source, there is no competitive exclusion in this model. Ultimately, the two populations should approach a stable steady state.

Figure 3 shows that the behavior of the caricature model is very similar to that of the full model, and therefore also fits the data reasonably well. Biologically, this means that one can account for the data by ignoring the competition between the truly $\mathrm{Tg} \mathrm{B}$ cells and the B cells from the diverse non- $\mathrm{Tg} /$ leaky- $\mathrm{Tg}$ repertoire. This model therefore provides the following novel interpretation of the data. Since the truly Tg B cells all compete for one subset of all epitopes they face a stronger intraspecific competition than non-Tg or leaky-Tg B cells do. That explains their poor competitive ability and their shorter lifetimes. Increasing the proportion of non- $\mathrm{Tg}$ cells in the bone marrow decreases the source of truly $\mathrm{Tg} \mathrm{B}$ cells, which relaxes their intraspecific competition for the survival signals recognized 

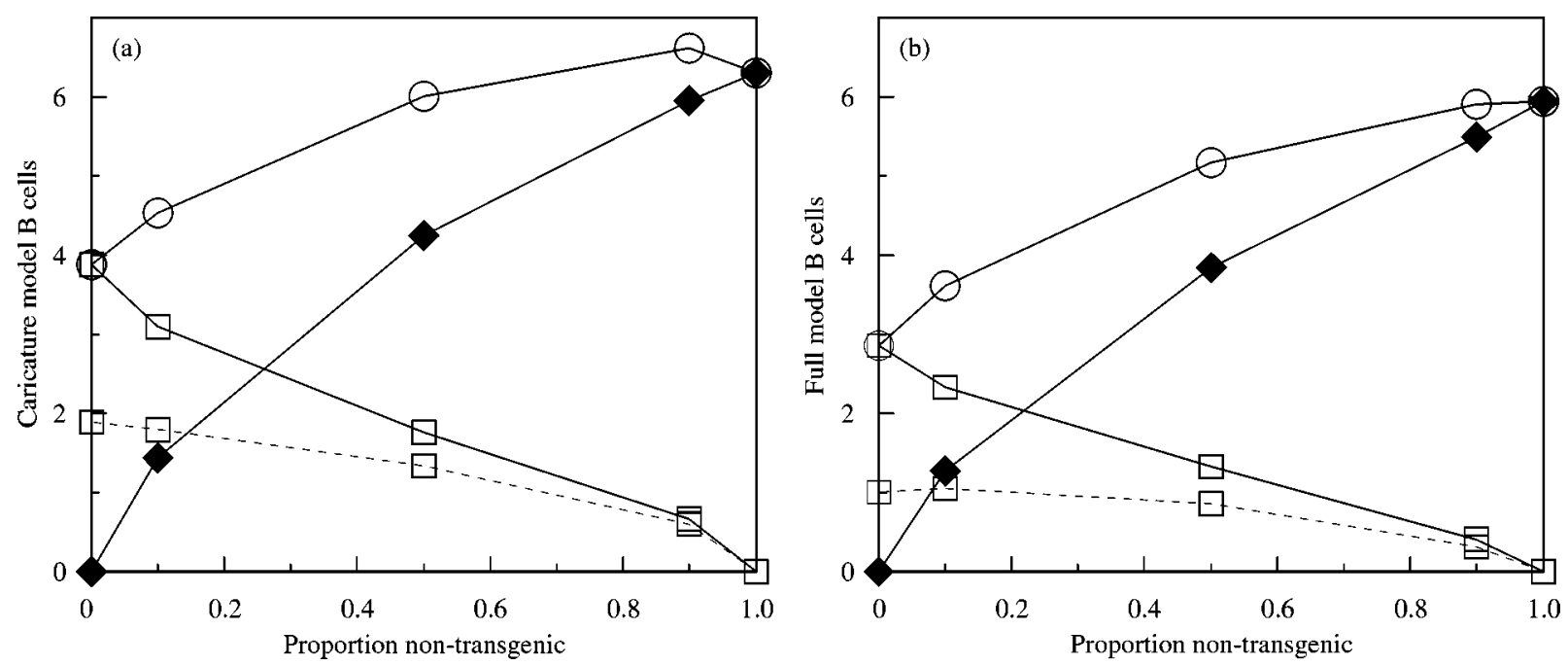

FIG 3. A comparison of the caricature model (in panel a) that is based upon intraspecific competition only and the data for the full model in panel (b). Panel (b) is identical to Fig. 1(c); for further details see the legend of Fig. 1. The total amounts of non-Tg and Tg B cells in panel (a) have to be calculated from the variables $B$ and $B_{0}$ in the caricature model. We use the same scaling as the full model by dividing the population size by the bone marrow output $M$. Further, the fraction $n_{l} /\left(n_{l}+n_{n}\right)$ of the total population $B$ corresponds to the leaky-Tg B cells. The remainder forms the non-Tg B cell population. Parameters as in Fig. 1 but $P_{L}=0.1$ throughout.

by truly Tg B cells. The truly Tg B cells therefore have the longest lifetimes when there are few truly Tg bone marrow precursors.

The fact that we can explain most of the data without invoking the postulated diversity-based competition between the non-Tg and the Tg repertoire of Freitas et al. (1995) and McLean et al. (1997), obviously does not mean that this cannot play any role. In fact, we now have a mechanistic interpretation for "diversity-based competition", i.e. a diverse repertoire utilizes more different resources and hence faces less intraspecific competition.

\section{A STRONG EXPERIMENTAL PREDICTION}

How can we determine if the model is correct in suggesting that most of the data can be explained with intraspecific competition? The model makes a strong prediction about the average lifetimes of truly $\mathrm{Tg}$ cells as a function of the truly $\mathrm{Tg}$ bone marrow precursors. The average truly $\mathrm{Tg} \mathrm{B}$ cell lifespan, as can be assessed by BrdU incorporation, should increase with the proportion of non-Tg B cell precursors in the bone marrow. Increasing the proportion of non-Tg B cell pre- cursors reduces the number of truly Tg cells and thus is expected to relax the intraspecific competition amongst the truly Tg B cells. The same experiment is, however, expected to intensify the inter-specific competitive effects of non-Tg B cells on truly Tg B cells. Thus, intraspecific and interspecific competition predict opposite effects on the BrdU incorporation of truly Tg B cells. Using RAG knock-out mice one could repeat the experiments in the absence of leaky-Tg B cells, and test the novel prediction on the truly $\mathrm{Tg} \mathrm{B}$ cell lifetimes.

\section{Conclusions}

The previous modeling study of these data (McLean et al., 1997) found evidence for interspecific competition by parameter fitting. Why are our results so different? Starting with a model for individual clones, and the different ligands they are competing for, we simplified this into a model that had the form of a conventional ecological model with density-dependent death rates. One difference is that the previous model (McLean et al., 1997) adopted a conventional term for competitive growth, and that we arrived 
at - similarly conventional-terms for competitive B cell survival. A second difference is that we lumped the leaky-Tg B cell clones with the non$\mathrm{Tg}$ population, rather than with the truly $\mathrm{Tg}$ clone (and consequently had to compute the total of $\mathrm{Tg} \mathrm{B}$ cells employing an unknown leakiness probability $P_{L}$ ). The main difference is, however, that the parameter fitting procedure of McLean et al. (1997) focused on estimating two interspecific competition parameters (i.e. the influence of the non- $\mathrm{Tg}$ repertoire on the $\mathrm{Tg}$ repertoire, and vice versa). By the structure of their model the intraspecific competition was based upon a shared parameter $K$ representing the resources both populations were competing for. Hence, pure intraspecific competition was not a possible outcome of their fitting procedure. Instead, we arrived at the conclusion that most competition is intraspecific by studying a model where the form of the competition emerges from clonal interactions for ligands providing survival signals. We found, rather than assumed, that most of the competition is intraspecific.

In retrospect, the interpretation of intraspecific competition was to be expected because competition for antigens is a form of resource competition (McLean et al., 1997; Freitas \& Rocha, 2000). Due to the lack of niche-differentiation, the intraspecific competition should always be stronger than the inter-specific competition between different species competing for resources. The novel interpretation of intraspecific competition is much simpler than the previous inter-specific interpretation because it only involves increased competition within the truly $\mathrm{Tg}$ clone when the fraction of Tg precursors increases. We no longer need to invoke a superior competitive ability of a diverse repertoire. It seems essential therefore to distinguish between these two forms of competition in interpreting these data. The modeling described in this paper prompted new experiments that are currently being carried out to test the novel interpretation.

In our model on the development of naive $B$ cell repertoires we have assumed that all the competition for B cell survival signals is antigen specific. Obviously, we cannot exclude that there is additional competition for non-specific factors, i.e. some form of global competition, regulating total B cell numbers. Global competition can be incorporated in our model by increasing every matrix element $K_{i j}$ with a fixed parameter defining the global influence that clone $j$ has on clone $i$. Thus, the structure of the full model would remain the same. In the mean field model this global influence will appear as an additional inter-specific competition term. We therefore expect our results to be robust to allowing for some form of non-specific competition.

Our main result is that these data can be interpreted largely in terms of the intraspecific competition within the truly $\mathrm{Tg}$ clone, and that alterations of the fraction of $\mathrm{Tg}$ cells in the bone marrow alter the intensity of the intraspecific competition. We therefore predict that the average turnover rates in the truly $\mathrm{Tg}$ clone should be positively correlated with the fraction of truly $\mathrm{Tg}$ cells in the bone marrow, rather than negatively correlated with the fraction of non-Tg bone marrow cells.

Portions of this work were performed under the auspices of the U.S. Department of Energy. This work was financially supported by NIH Grant AI28433, NATO Grant GRC960019, the Theoretical Immunology program of the Santa Fe Institute funded by the Joseph P. and Jeanne M. Sullivan Foundation, and by ARC, ANRS MNERT and Sidaction Grants.

\section{REFERENCES}

Agenes, F., Rosado, M. M. \& Freitas, A. A. (1997). Independent homeostatic regulation of B cell compartments. Eur. J. Immunol. 27, 1801-1807.

De Boer, R. J. \& Perelson, A. S. (1994). T cell repertoires and competitive exclusion. J. theor. Biol. 169, 375-390.

De Boer, R. J. \& Perelson, A. S. (1995). Towards a general function describing T cell proliferation. J. theor. Biol. 175, 567-576.

De Boer, R. J. \& Perelson, A. S. (1997). Competitive control of the self-renewing $\mathrm{T}$ cell repertoire. Int. Immunol. 9, 779-790.

Ernst, B., Lee, D. S., Chang, J. M., Sprent, J. \& Surh, C. D. (1999). The peptide ligands mediating positive selection in the thymus control $\mathrm{T}$ cell survival and homeostatic proliferation in the periphery. Immunity 11, 173-181.

Freitas, A. A. \& Rocha, B. B. (1993). Lymphocyte lifespans: homeostasis, selection and competition. Immunol. Today 14, 25-29.

Freitas, A. A. \& Rocha, B. (2000). Population biology of lymphocytes: the flight for survival. Annu. Rev. Immunol. 18, 83-111.

Freitas, A. A., Rosado, M. M., Viale, A. C. \& Grandien, A. (1995). The role of cellular competition in B cell survival and selection of B cell repertoires. Eur. J. Immunol. 25, 1729-1738. 
Freitas, A. A., Agenes, F. \& Coutinho, G. C. (1996). Cellular competition modulates survival and selection of $\mathrm{CD}^{+}$T cells. Eur. J. Immunol. 26, 2640-2649.

Goodnow, C. C., Crosbie, J., Adelstein, S. Lavoie, T. B., Smith-Gill, S. J., BrinK, R. A. et al. (1988). Altered immunoglobulin expression and functional silencing of self-reactive B lymphocytes in transgenic mice. Nature 334, 676-682.

KiePER, W. C. \& JAmeson, S. C. (1999). Homeostatic expansion and phenotypic conversion of naive T cells in response to self peptide/MHC ligands. Proc. Natl Acad. Sci. U.S.A. 96, 13306-13311.

Mackall, C. L. \& GRess, R. E. (1997). Thymic aging and T-cell regeneration. Immunol. Rev. 160, 91-102.

Mackall, C. L., Bare, C. V., Granger, L. A., Sharrow, S. O., Titus, J. A. \& Gress, R. E. (1996). Thymic-independent $\mathrm{T}$ cell regeneration occurs via antigen-driven expansion of peripheral $\mathrm{T}$ cells resulting in a repertoire that is limited in diversity and prone to skewing. J. Immunol. 156, 4609-4616.

Mackall, C. L., HaKim, F. T. \& GRess, R. E. (1997). T-cell regeneration: all repertoires are not created equal. Immunol. Today 18, 245-251.

McLean, A. R., Rosado, M. M., Agenes, F., VasconcelLOS, R. \& FreITAS, A. A. (1997). Resource competition as a mechanism for B cell homeostasis. Proc. Natl Acad. Sci. U.S.A. 94, 5792-5797.

Press, W. H., Plannery, B. P., Teukolsky, S. A. \& Vetterling, W. T. (1988). Numerical Recipes in C. The Art of Scientific Computing. Cambridge: Cambridge University Press.

Rocha, B., Dautigny, N. \& Pereira, P. (1989). Peripheral T lymphocytes: expansion potential and homeostatic regulation of pool sizes and CD4/CD8 ratios in vivo. Eur. J. Immunol. 19, 905-911.

Rosado, M. M. \& Freitas, A. A. (1998). The role of the $\mathrm{B}$ cell receptor V region in peripheral B cell survival. Eur. J. Immunol. 28, 2685-2693.

Rusconi, S. \& Kohler, G. (1985). Transmission and expression of a specific pair of rearranged immunoglobulin $\mu$ and $\kappa$ genes in a transgenic mouse line. Nature 314, 330-334.

Surh, C. D. \& Sprent, J. (2000). Homeostatic T Cell Proliferation. How far can $t$ cells be activated to selfligands? J. Exp. Med. 192, 0.

TANCHOT, C. \& Rocha, B (1995). The peripheral T cell repertoire: independent homeostatic regulation of virgin and activated $\mathrm{CD}^{+} \mathrm{T}$ cell pools. Eur. J. Immunol. 25, 2127-2136.

TANCHOT, C. \& RochA, B. (1997). Peripheral selection of $\mathrm{T}$ cell repertoires: the role of continuous thymus output. J. Exp. Med. 186, 1099-1106.
TANCHOT, C. \& RochA, B. (1998). The organization of mature T-cell pools. Immunol. Today 19, 575-579.

Viret, C., Wong, F. S. \& Janeway JR, C. A. (1999). Designing and maintaining the mature TCR repertoire: the continuum of self-peptide:self-MHC complex recognition. Immunity 10, 559-568.

Witherden, D., VAN Oers, N., Waltzinger, C., Weiss, A., Benoist, C. \& Mathis, D. (2000). Tetracycline-controllable selection of CD4( $\left.{ }^{+}\right)$T cells: half-life and survival signals in the absence of major histocompatibility complex class II molecules. J. Exp. Med. 191, 355-364.

\section{APPENDIX}

The form of the B cell population dynamics in the mean field model is

$$
\frac{\mathrm{d} B}{\mathrm{~d} t}=m-\mathrm{d} B-0.5 d \gamma B^{2},
$$

where $m=n_{n}+n_{l}$ and $\gamma=c / e<0$ for the non$\mathrm{Tg} /$ leaky-Tg population, and $m=M-n_{n}-n_{l}$ and $\gamma=1$ for the truly $\mathrm{Tg}$ clone. The unique positive steady state of this equation is at

$$
\bar{B}=\frac{1}{\gamma}\left(\frac{\sqrt{d^{2}+2 d m \gamma}}{d}-1\right) .
$$

Since $\sqrt{d^{2}+2 d \gamma m} / d>1$, the steady state $\bar{B}$ is indeed strictly positive. In our model the death rate $d$ in the absence of competition is small, i.e., $d=0.001 /$ day. The $d^{2}$ term in eqn (A.2) is therefore vanishingly small. For $m=500$ and the non-Tg $\gamma=0.1$, eqn (A.2) yields $\bar{B}=3152$. For the truly $\operatorname{Tg} \gamma=1$ this yields $\bar{B}=999$. Scaling the population sizes by the total source $m=500$ this corresponds to $\bar{B}=6.3$, and $\bar{B}=2$, respectively. The former number can be found in Fig. 3(b) at $100 \%$ non-Tg cells. 